\title{
Best and Worst External Viewpoints for Teleoperation Visual Assistance
}

\author{
Mahmood M. Shilleh \\ Computer Science \& Engineering \\ Texas A\&M University \\ College Station, Texas USA \\ mahmoods@tamu.edu \\ Jan Dufek \\ Computer Science \& Engineering \\ Texas A\&M University \\ College Station, Texas USA \\ dufek@tamu.edu
}

\author{
Qusai A. Amer \\ Computer Science \& Engineering \\ Texas A\&M University \\ College Station, Texas USA \\ qusai3mer@tamu.edu \\ Robin R. Murphy \\ Computer Science \& Engineering \\ Texas A\&M University \\ College Station, Texas USA \\ robin.r.murphy@tamu.edu
}

\begin{abstract}
A HRI study with 31 expert robot operators established that an external viewpoint from an assisting robot could increase teleoperation performance by $14 \%$ to $58 \%$ while reducing human error by $87 \%$ to $100 \%$ This video illustrates those findings with a side-by-side comparison of the best and worst viewpoints for the passability and traversability affordances. The passability scenario uses a small unmanned aerial system as a visual assistant that can reach any viewpoint on the idealized hemisphere surrounding the task action. The traversability scenario uses a small ground robot that is restricted to a subset of viewpoints that are reachable.
\end{abstract}

\section{CCS CONCEPTS}

- Robotics • Perception • Machine Learning • Interaction Design

KEYWORDS: Robotics; User/Machine Systems; Distributed Intelligence; Image Processing and Computer Vision

\section{ACM Reference format:}

Mahmood M. Shilleh, Qusai Amer, Jan Dufek, and Robin Murphy. 2021. Best and Worst External Viewpoints for Teleoperation Visual Assistance. In the Companion of the 2021 ACM/IEEE International Conference on HumanRobot Interaction (HRI'21 Companion), March 8-11, 2021, Boulder, CO, USA. ACM, NY, NY, USA. 2 pages. https://doi.org/10.1145/3434074.3447280

\section{Introduction}

It is common practice in field robotics, such as disaster response and nuclear decommissioning, to use two robots for a perceptually difficult task, e.g., opening a door or reaching into narrow and irregular voids. A secondary robot, dubbed a visual assistant, gives

\footnotetext{
Permission to make digital or hard copies of all or part of this work for personal or classroom use is granted without fee provided that copies are not made or distributed for profit or commercial advantage and that copies bear this notice and the full citation on the first page. Copyrights for third-party components of this work must be honored. For all other uses, contact the Owner/Author. HRI '21 Companion, March 8-11, 2021, Boulder, CO, USA (c) 2021 Copyright is held by the owner/author(s).

ACM ISBN 978-1-4503-8290-8/21/03. https://doi.org/10.1145/3434074.3447280
}

a teleoperator a better external view of the task than what is seen from the onboard cameras or ranging system on the primary robot. As a result, task time and human error is reduced. Unfortunately, there are issues with the current state of practice. It increases the cognitive load on the operator having to control two robots. Additionally, studies [1,2] indicate that while teleoperators prefer being able to choose the viewpoint provided by the assistant, they do not necessarily pick the viewpoint that maximizes their performance and minimizes errors. Furthermore, even if the optimal viewpoint was known, there is no guarantee that the assisting robot could reach it, especially in a cluttered environment.

A recent HRI study [3] learned a quantitative ranking of possible viewpoints for four different visual affordances. The study engaged 31 expert robot teleoperators from the US and Japan with expertise in civilian or military bomb squad robotics or in nuclear decommissioning. The study produced rankings of viewpoints for perceptual affordances for four different tasks: passability, traversability, manipulability, and reachability. If the visual assistant was located at the highest ranked viewpoint for that affordance, teleoperation performance was increased by $14 \%$ to $58 \%$ while reducing human error by $87 \%$ to $100 \%$.

However, that study was conducted and evaluated with a high-fidelity computer-based simulation, not in the physical world. This video illustrates the real-world applicability of the findings by staging demonstrations in the physical world, offering side-by-side comparisons of what the best and worst views for an affordance look like.

\section{Underlying Theory}

The video briefly reviews the underlying theory for visual assistance, which is given in more detail here. Prior work by [4] demonstrated that Gibsonian visual affordances could be associated with tasks. For example, many tasks have a component where the relative distance to an object must be estimated, called reachability. Other tasks require a robot to position itself and end effector in the right configuration to manipulate an object, i.e., 
manipulability. [3] extended this concept, representing the space of viewpoints for a particular affordance as a hemisphere. The ranked value of the viewpoints can be derived from machine learning, using agglomerative hierarchical cluster analysis with average linages, and that adjacent viewpoints with similar ratings can be clustered into manifolds of viewpoints with equivalent value. The ranked manifolds for a hemisphere are projected onto two-dimensional, circle areas referred to as hemispherical, or manifold, maps. Fig. 1 shows the manifold maps for passability, traversability, manipulability, and reachability. Note that the relative value of manifolds is shown as red (not helpful), yellow (borderline), green (helpful).

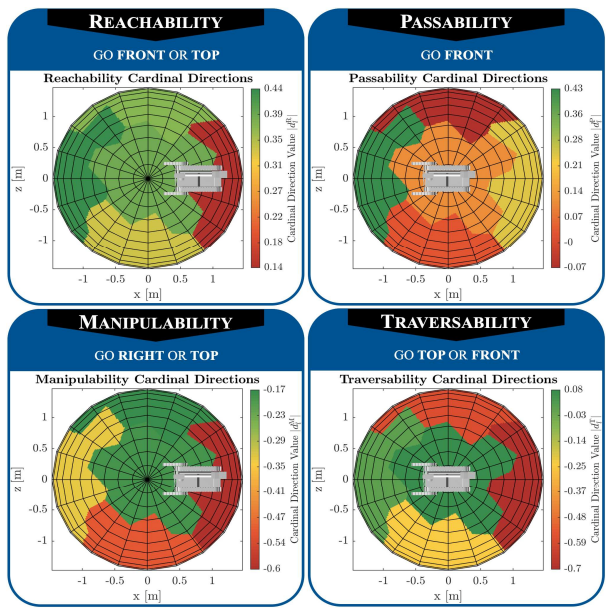

Figure 1: Detailed Hemispherical Maps

\section{Visual Assistance from an Aerial Vehicle for Passability}

The passability scenario simulates a primary robot (Endeavor Packbot 510) navigating through a narrow doorway with the help of an aerial vehicle. The visual assistant is nominally a Fotokite tethered unmanned aerial system, but simulated with a camera on a tripod due to equipment problems. The video shows side-by-side, the video from the camera at the locations which were predicted to be the best (in front and slightly above the Packbot) and worst (at either side) viewpoints for passability. See Fig. 2.

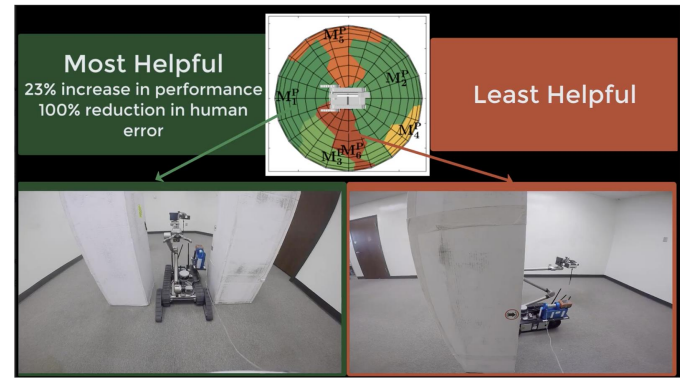

Figure 2: UAS Assistant Best and Worst (Passability Affordance)

\section{Visual Assistance from a Ground Robot for Traversability}

The traversability scenario simulates a primary robot (Endeavor Packbot 510) traversing two narrow rails with the help of a small ground vehicle (Endeavor First Look). Unlike the Fotokite, the First Look cannot reach the optimal viewpoint for traversability, which would be above the action, but instead is located at the best viewpoint it can reach. While this viewpoint is not as good as can be imagined from an aerial vehicle, it is clearly better than the worst viewpoint (behind). See Fig. 3. Given the least helpful view, the video shows that the operator makes a critical mistake with the Packbot by falling off the path. When given the most helpful view, the operator easily traverses the path.

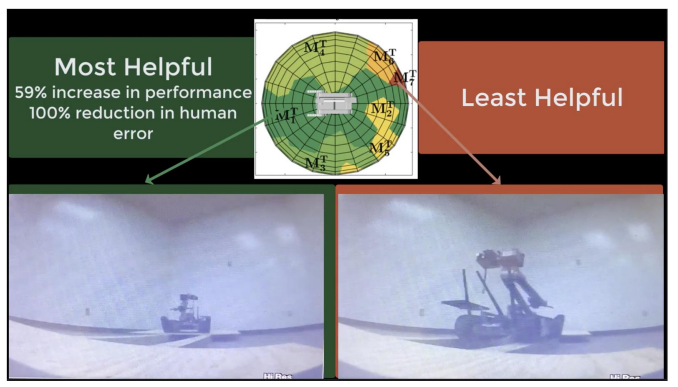

Figure 3: UGV Assistant Best and Worst (Traversability Affordance)

\section{Conclusions}

This video illustrates the distinct visual differences between viewpoints as predicted by (Dufek 2020). It is not intended as a formal confirmation of the theory, only as a qualitative demonstration of two of the four affordances. The obvious differences in viewpoints coupled with the statistically significant increase in performance and reduction of human error in the simulator study argues for the merit of visual assistants for reducing cognitive workload in human-robot interaction.

\section{ACKNOWLEDGMENTS}

This material is based upon work supported by the Department of Energy and National Science Foundation under Grants DEEM0004483 and IIS-1945105. Any opinions, findings, and conclusions or recommendations expressed in this material are those of the author(s) and do not necessarily reflect the views of DOE or NSF.

\section{REFERENCES}

[1] G. T. McKee, B. G. Brooks and P. S. Schenker. 2003. Human-robot interaction for intelligent assisted viewing during teleoperation. In Proceedings of the 36th Annual Hawaii International Conference on System Sciences, January 6 - 9, 2003, Big Island, HI. 10.1109/HICSS.2003.1174286

[2] R. R. Murphy. 2015. Meta-analysis of Autonomy at the DARPA Robotics Challenge Trials. f. Field Robot 32, 2 (March 2015), 189-191. https://doi.org/10.1002/rob.21578

[3] Jan Dufek. 2020. Best Viewpoints for External Robots or Sensors Assisting Other Robots. Ph.D. Dissertation. Texas A\&M University, College Station, TX.

[4] A. M. Morison, T. Murphy, and D. D. Woods. 2016. Seeing Through Multiple Sensors into Distant Scenes: The Essential Power of Viewpoint Control. In Human-Computer Interaction. Interaction Platforms and Techniques, June 19, 2016, Springer International Publishing, 388-399. https://doi.org/10.1007/978-3-319-39516-6_37 